\title{
High-efficiency generation of nanomaterials via laser ablation synthesis in solution with in-situ diagnostics for closed-loop control
}

Freeland, Brian, McCann, Ronan, Foley, Greg, Brabazon, Dermot

Brian Freeland, Ronan McCann, Greg Foley, Dermot Brabazon, "Highefficiency generation of nanomaterials via laser ablation synthesis in solution with in-situ diagnostics for closed-loop control," Proc. SPIE 11269, Synthesis and Photonics of Nanoscale Materials XVII, 112690M (2 March 2020); doi: $10.1117 / 12.2545878$

SPIE. Event: SPIE LASE, 2020, San Francisco, California, United States 


\title{
High-efficiency generation of nanomaterials via laser ablation synthesis in solution with in-situ diagnostics for closed-loop control
}

\author{
Brian Freeland ${ }^{\mathrm{a}, \mathrm{b}, \mathrm{c}, \mathrm{d}}$, Ronan McCann ${ }^{* a, b, c}$, Greg Foley ${ }^{\mathrm{a}, \mathrm{d}}$, Dermot Brabazon ${ }^{\mathrm{a}, \mathrm{b}, \mathrm{c}}$ \\ ${ }^{a}$ Advanced Processing Technology Research Centre, Dublin City University, Glasnevin, Dublin 9,

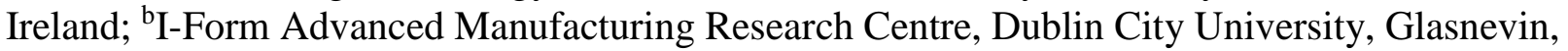 \\ Dublin 9, Ireland; ${ }^{\mathrm{C}}$ National Centre for Plasma Science and Technology, Dublin City University, \\ Glasnevin, Dublin 9, Ireland; 'S School of Biotechnology, Dublin City University, Glasnevin, Dublin \\ 9, Ireland
}

\begin{abstract}
Driven by an ever-increasing demand for nanomaterials with specific functionalities, physical synthesis techniques such as Laser Ablation Synthesis in Solution (LASiS) have gained significant interest over in recent years. Commercial wet chemical synthesis methods, while having significantly higher nanomaterial yields than LASiS, typically have considerable negative environmental impact through the use of harmful reagents and solvents. LASiS therefore represents a route towards the sustainable "green" production of nanomaterials however the significant challenge to its commercialization is that of comparably low nanomaterial yields. Significant effort has been made towards increasing the production rates of LASiS, however many of the reported advances have relied on the use of high power $(>20 \mathrm{~W})$ or short pulse $(<10 \mathrm{ps})$ laser systems which have high capital costs. Other advances have examined moving from batch production in small volumes towards the use of continuous production through the use of solvent flow systems.

Combining these advances, we have developed a new system for nanomaterial generation via LASiS incorporating a low cost, low power $(<4 \mathrm{~W}) \mathrm{Nd}$ :YAG laser and solvent flow system for high-efficiency nanomaterial generation. This study has shown an increase in productivity from $2.5 \pm 0.5 \mathrm{mg} / \mathrm{hr}$ for an $11 \mathrm{~mL}$ batch colloid, to continuous production yields of $17 \pm 0.7 \mathrm{mg} / \mathrm{hr}$ under flow conditions.
\end{abstract}

Keywords: Laser Ablation Synthesis in Solution, LASiS, Pulsed Laser Ablation in Liquid, PLAL, Nanoparticles, Dynamic Light Scattering, Laser Ablation,

\section{INTRODUCTION}

Throughout the previous decade, many advancements have been made in the field of nanomaterial synthesis via Laser Ablation Synthesis in Solution (LASiS). In this technique, a solid target is immersed in a liquid solvent and subjected to high power pulsed laser irradiation ${ }^{1-3}$. This irradiation causes material ablation, with the ejected material forming a nanoparticle colloid in the solvent. This physical technique is highly sustainable due to the ability to produce nano-colloids with are ligand-free and produced without the need for environmentally harmful reagents used in wet-chemical production methods. This technique also allows for a range of single-step post-production functionalizations enabling their use in areas such as chemical separation, biosensing, cellular labelling, display technology. Despite these advances, the challenge of production scale-up and transfer from batch to continuous production has yet to be fully realized and is a necessary step for this technique to compete commercially with wet-chemical production.

Here, we examined the synthesis of silicon nanoparticles via LASiS using a low-power solid-state Nd:YAG laser over various power ranges to study the effect on nanoparticle production. Nanoparticle size, size dispersion and ablation rate were studied via Dynamic Light Scattering (DLS). Initial steps towards production scale-up is examined utilizing an initial continuous flow during ablation prototype system with online DLS, with the continuous flow regime was compared to the standard static-flow batch processing with nanoparticle yield and quality examined.

*ronan.mccann@i-form.ie; phone +353 1700 7631; fax +353 1700 7148; i-form.ie

Synthesis and Photonics of Nanoscale Materials XVII, edited by Jan J. Dubowski,

David B. Geohegan, Andrei V. Kabashin, Proc. of SPIE Vol. 11269, 112690M

(c) 2020 SPIE · CCC code: 0277-786X/20/\$21 · doi: 10.1117/12.2545878

Proc. of SPIE Vol. 11269 112690M-1 


\section{EXPERIMENTAL SETUP}

\subsection{Laser System and Nanoparticle Generation}

In this paper, a picosecond-pulsed diode-pumped solid state Nd:YAG laser (WEDGE HF 1064; BrightSolutions; Italy; $\lambda$ $=1064 \mathrm{~nm} ; \tau_{\mathrm{p}}=700 \mathrm{ps} ; f_{\mathrm{PRF}}=10 \mathrm{kHz}$ ) was used to produce nanoparticle colloids. A two-dimensional scanning galvanometer (SS-12; Raylase, Germany) was used to raster the beam in the xy-plane and a one-dimensional movable stage (404-3PD; PI, Germany) was used to control the position of the target in the beam waist. The laser beam was at a speed of $2 \mathrm{~mm} / \mathrm{s}$ moving in an Archimedean spiral pattern over the top surface of the target using a beam diameter of 103 $\mu \mathrm{m}$.

The target material used for all tests was silicon rod (99.998\% metals basis; Alfa Aesar, UK) with a diameter of $8.28 \mathrm{~mm}$ and thickness of $6 \mathrm{~mm}$. For batch production (Figure 1a), the target was immersed in either $6 \mathrm{~mL}$ and $11 \mathrm{~mL}$ of ultrapure DI water in two different glass vials such that there was $4 \mathrm{~mm}$ of DI water above the top of the target. For continuous liquid flow, the target was mounted within the center of the flow reactor cell, illustrated in Figure 1b. The flow reactor was designed to ensure laminar flow conditions in order to reduce the effects of the cavitation bubble ${ }^{4}$, plume occlusion ${ }^{5}$ and particle aggregation at the ablation site ${ }^{6,7}$. Continuous flow was maintained at rate $100 \mathrm{~mL} / \mathrm{min}$. A Dynamic Light Scatting (DLS) particle sizer was integrated to the flow system to allow for particle quality measurements during production

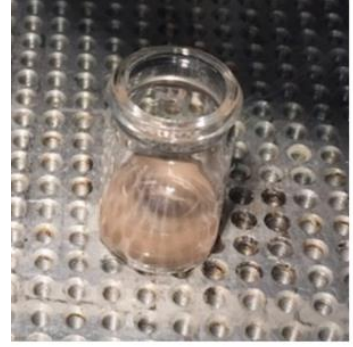

(a)

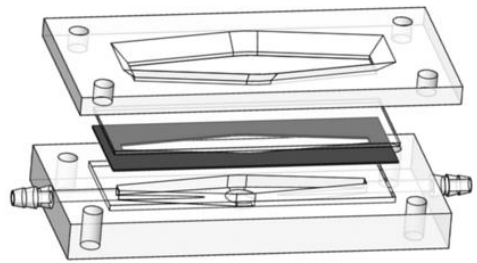

(b)

Figure 1. (a). $6 \mathrm{ml}$ batch LASiS production glass vial and (b) continuous LASiS production flow cell.

\subsection{Metrology}

Nanoparticle morphology was examined using both transmission election microscopy (TEM) and DLS techniques. The TEM used was a FEI Titan with Field Emission Gun and spherical aberration corrector system (Cs-corrector) of the objective lens operated at $300 \mathrm{kV}$. Analysis was performed using a copper mesh TEM grid with $40 \mu \mathrm{L}$ of sample applied and allowed to evaporate at room temperature. The DLS used was a NanoFlex (Microtrac, USA) and was used both for offline tests post-ablation, and online testing during continuous prodution. The optical properties of the produced nanoparticles were characterized using UV-Vis-NIR spectroscopy (Cary 50; Varian, USA), with a scanning range of 200 $-800 \mathrm{~nm}$ at a rate of $20 \mathrm{~nm} / \mathrm{s}$ with solvent background correction applied.

\section{RESULTS AND DISCUSSION}

\subsection{Batch Production}

A SiNP colloid was synthesized using $1064 \mathrm{~nm}$ wavelength irradiation at a laser fluence of $1.83 \mathrm{~J} / \mathrm{cm}^{2}$ for 10 -minute ablation. The nanoparticle size and morphology were analyzed via DLS and TEM respectively, shown in Figure 2. TEM revealed that the produced nanoparticles are spherical with a peak diameter of approximately $80 \mathrm{~nm}$. DLS showed a nanoparticle size distribution ranging from $10 \mathrm{~nm}-160 \mathrm{~nm}$, with the distribution peaking at $90 \mathrm{~nm}$. The disparity between the TEM and DLS measurements is due to the hydrodynamic diameter of the nanoparticles in suspension being larger than that of their true, dry diameter. Though DLS is a fast, non-destructive technique, this highlights the continuing need for TEM analysis for any studies involving nanoparticles. The particle size distribution is similar to that produced via LASiS using a femtosecond Ti:Sapphire laser $\left(\tau_{\mathrm{p}}=110 \mathrm{fs} ; \lambda=800 \mathrm{~nm}\right)^{8}$. 

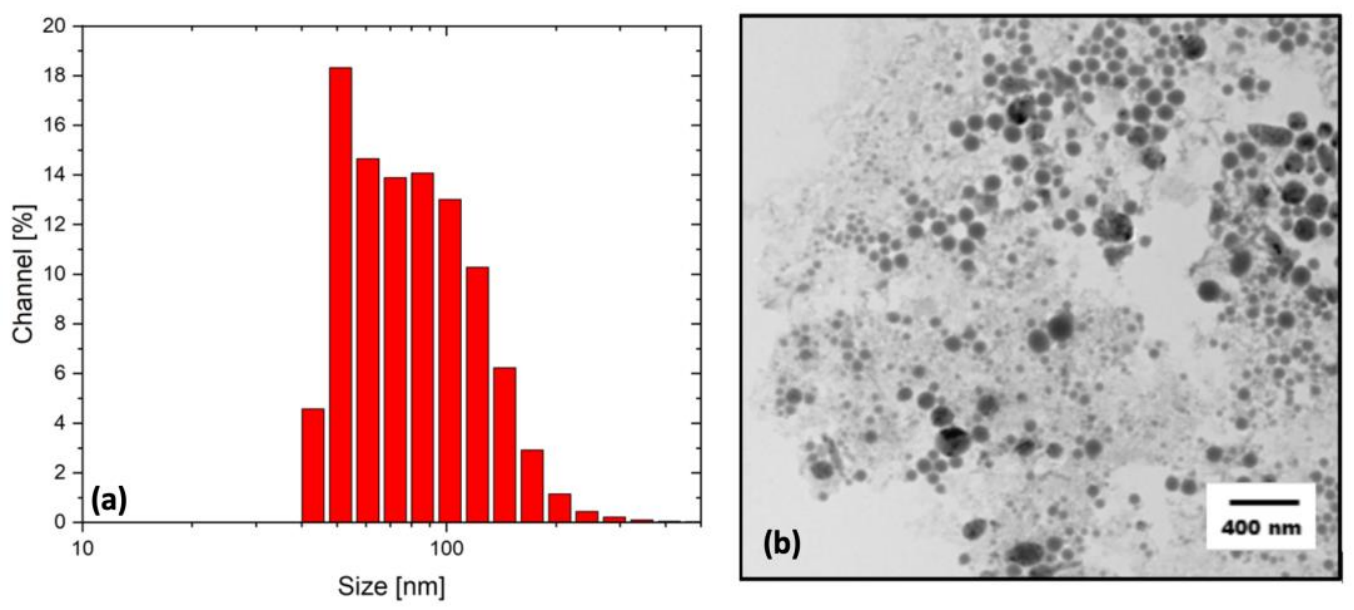

Figure 2. (a) DLS nanoparticle size distribution ( $90 \mathrm{~nm} \pm 70 \mathrm{~nm}$ ), (b) TEM images of SiNPs with scale bar of $400 \mathrm{~nm}$ generated using $1064 \mathrm{~nm}$ batch laser irradiation at a fluence of $1.83 \mathrm{~J} / \mathrm{cm} 2$.

The effect of fluence on ablation rate in the batch production regime $(11 \mathrm{~mL}$ solvent) was determined under 5-minute ablation trials (Figure 3a). The ablation rate increased linearly with fluence, but with a high average process variation (24 $\%$ ) within the tests. The linear fit allowed for the target fluence threshold to be determined as $0.28 \mathrm{~J} / \mathrm{cm}^{2}$, however given the large process error bars and low fit correlation (Adjusted $\mathrm{R}^{2}=0.85$ ), this should be taken as an estimate. The UV-Vis absorbance spectra of the collected colloids were in alignment with the gravimetrical measurements of NPs production.
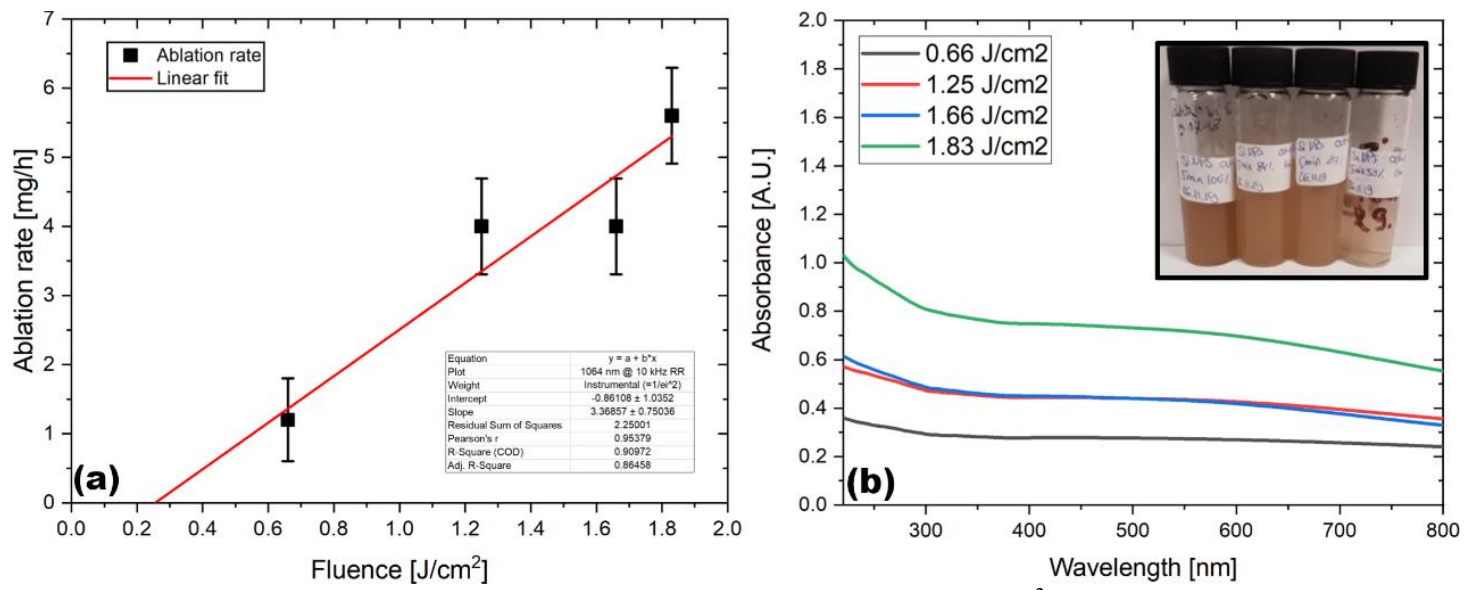

Figure 3. (a) Ablation rate for 5-minute tests up to the maximum fluence of $1.83 \mathrm{~J} / \mathrm{cm}^{2}$, (b) the UV-Vis absorbance spectra illustrating the effect of laser fluence on colloid concentration and (b inset) the corresponding colloids produced (fluence descending left to right).

Time dependence productivity of batch production was investigated, ablating silicon targets at time intervals from 3 mins to 20 mins (shown in Figure 4). The ablation rate was calculated from gravimetric measurements of the targets pre- and post-ablation. Initial tests were performed with a $6 \mathrm{~mL}$ batch volume, resulting in a logarithmic decline in the ablation rate over the period examined. A initial ablation rate of $7.8 \mathrm{mg} / \mathrm{hr}$ was seen to reduce to $1.7 \mathrm{mg} / \mathrm{hr}$ after 20 minutes. This decline is indicative of particle occlusion at the ablation site $^{9}$, reducing the laser fluence at the target surface, in agreement with the Beer-Lambert Law. In order to mitigate this, a larger volume test was carried out with $11 \mathrm{~mL}$ of solvent. The final productivity was increased to $2.5 \pm 0.5 \mathrm{mg} / \mathrm{hr}$ due to a lower concentration of occluding particles, however the logarithmic decline was still observed. It's clear from these results that simply by increasing the batch volume would not produce a scalable, steady-state production process necessary for larger scale production. 


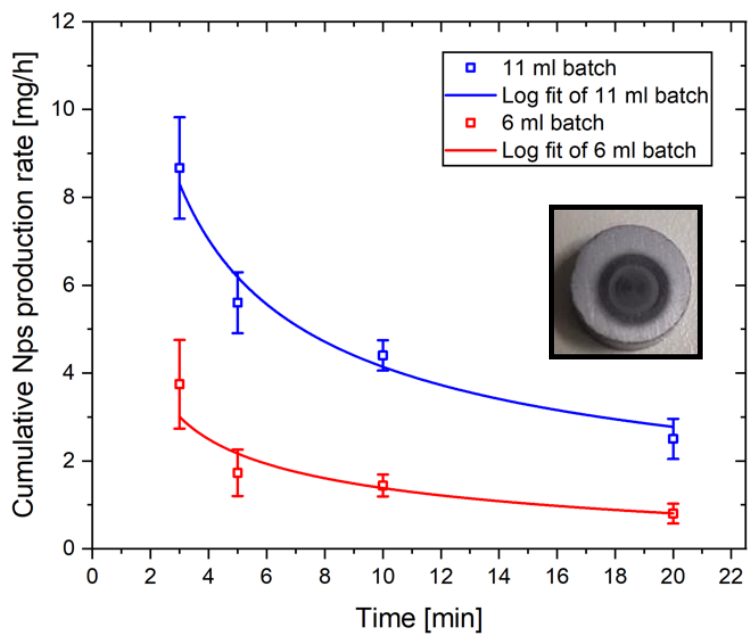

Figure 4. Cumulative ablation rate of $6 \mathrm{~mL}$ and $11 \mathrm{~mL}$ batch production using a $1064 \mathrm{~nm}$ laser and (inset) image of the ablation crater and heat effected zone on the target surface after a 20 -minute ablation.

\subsection{Production Scale-up via Continuous Production}

Dynamic-flow laser ablation of the target allows for continuous colloid production, with nanoparticle colloid volumes only limited by target material availability and laser operating time. Continuous production offers the promise of removing occluding NPs from the ablation site, mitigating the absorption-related problems discussed with batch production, promising higher yields and a steady-state production process. A description of the continuous production process developed is shown in Figure 5. Initial ablation tests were carried out for 20 minutes runs in triplicate.

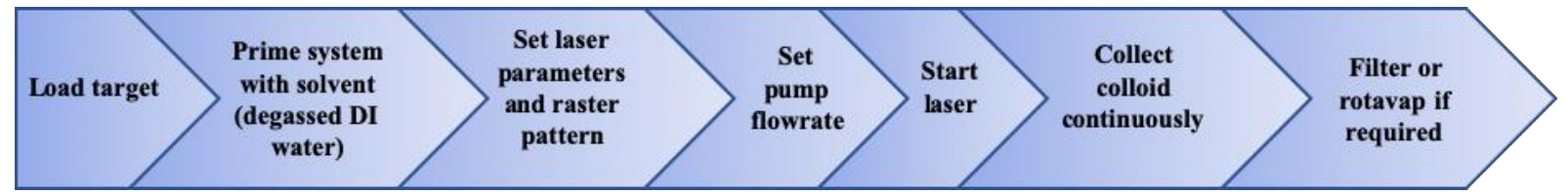

Figure 5. Process flow diagram of the continuous production process.

The size distribution and absorbance spectra of the SiNPs colloids produced are displayed in Figure 6. The size distribution (Figure 6a) revealed nanoparticle similar to those produced via batch production, with nanoparticle diameters of $140 \mathrm{~nm}$ $\pm 65 \mathrm{~nm}$, a spherical morphology was observed in Figure 7b. The larger peak diameter can be attributed the continuously produced particles not undergoing "zero-dimensional ablation" 10 whereby NPs in the batch colloid can interact with the laser irradiation and possibly fragment into smaller diameters NPs. The optical absorbance spectrum (Figure 6b) of the dynamically produced colloid was comparable with that produced via static ablation with no new spectral features present, indicative of similar quality nanoparticles. The continuous process resulted in a much higher yield of nanoparticles (Figure 7a) than that of batch production. 

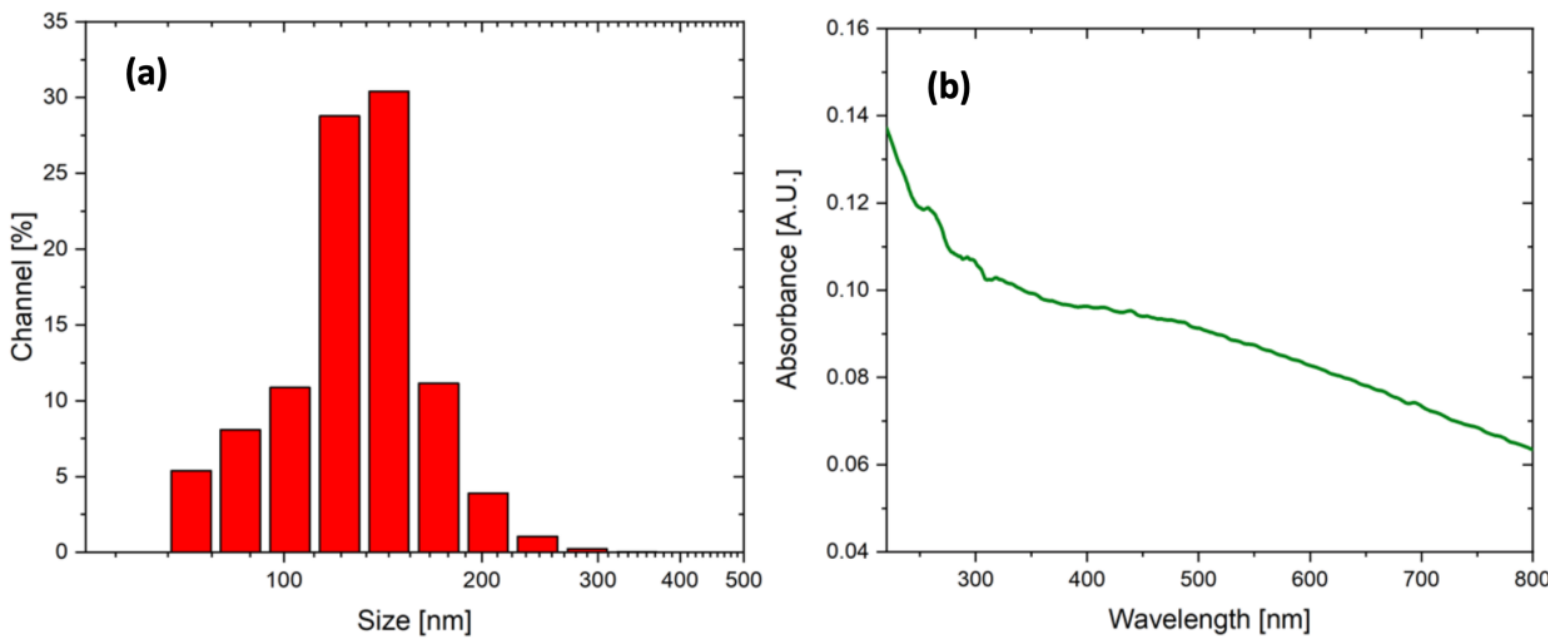

Figure 6. Nanoparticle size distribution $(140 \pm 65 \mathrm{~nm}$ ) (a) and optical absorbance spectrum (b) of continuous flow synthesized SiNPs.
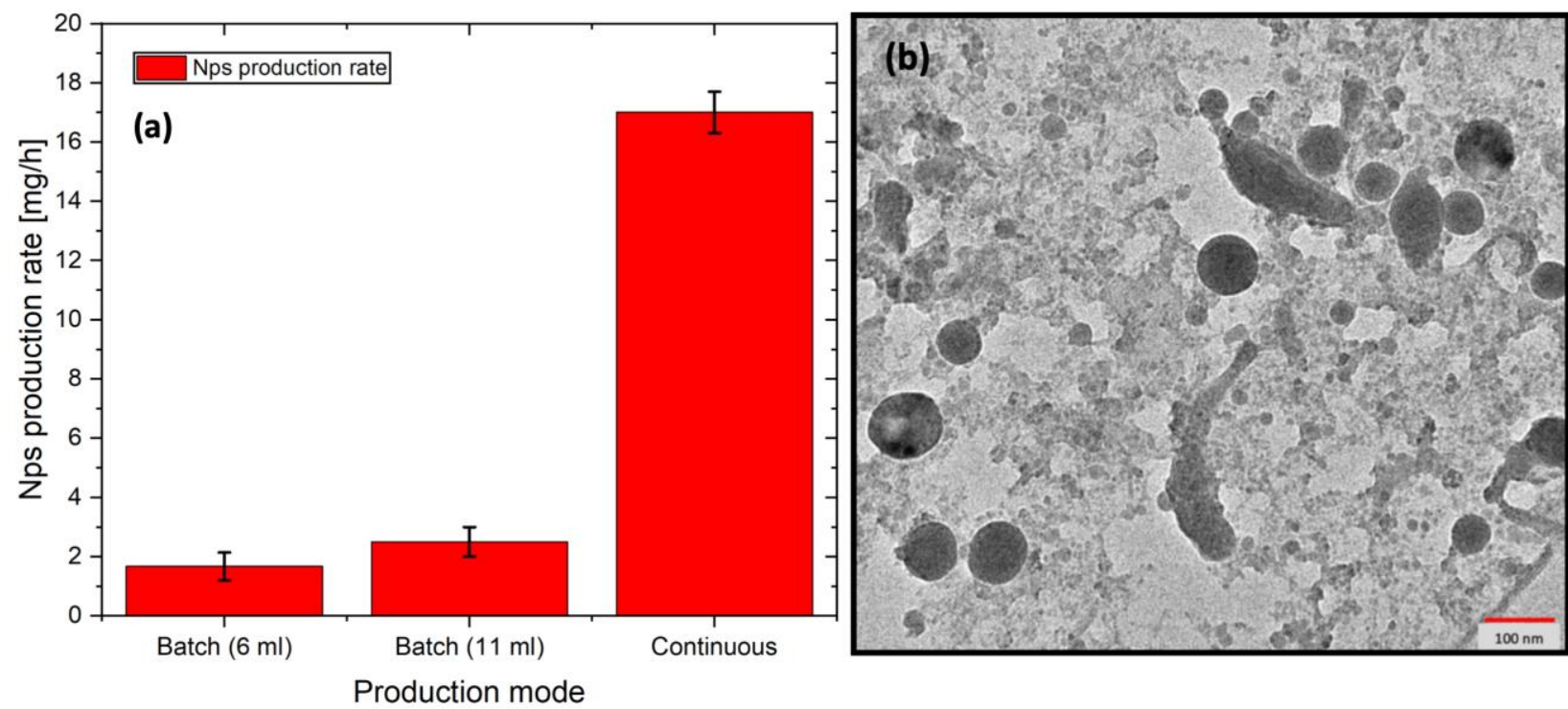

Figure 7. Per-hour production rates extrapolated from 20-minute tests for batch and continuous (a) and (b) TEM image of SiNPs produced via continuous LASiS with $100 \mathrm{~nm}$ scale bar.

For 20-minute ablations, batch production was seen to generate NP yields of $2.5 \pm 0.5 \mathrm{mg} / \mathrm{hr}$ within a colloid volume of $11 \mathrm{~mL}$ while the continuous process resulted in yields of $17 \pm 0.7 \mathrm{mg} / \mathrm{hr}$ in $1000 \mathrm{~mL}$ of colloid, showing lower production process variation. Although the resulted colloid was lower in concentration due to the high rate of liquid flow, with development it should be possible to increase the concentration through the use of re-circulation of the colloid during ablation (in a "semi-batch" mode) prior to collection and filtering. The increased ablation efficiency and relative uniformity of the continuous ablated target surface demonstrates that the occluding material such as particles and the generated plume were significantly by-passed during ablations, signifying that the flow cell design shows promise for future work. The integration of online particle sizing also shows promise for rapid optimisation and process monitoring and closed-loop control. 


\section{CONCLUSIONS}

We have presented the first steps towards the development of an in-house, continuous flow system for nanoparticle colloid production. The results suggest that appropriate selection of laser conditions, such as fluence, for a given material can lead to highly optimized NP production. Under continuous production, similar NP size and morphology was seen comparable to that produced via a batch technique, however, larger variations in peak size, size distribution were seen due to slight variation in the ablation conditions. Ultimately, the continuous flow ablation regime showed promise in producing large, scalable volumes of nanoparticles more efficiently than batch production. Also examined was the integration of particle sizing via online DLS measurements, allowing for real-time process monitoring and the future ability for closed-loop control. Though further work is required to improve qualities such as colloid concentration, the process potential tremendous promise towards increasing ablation efficiency with existing lasers and towards realizing high yield laser generated industrial-scale nanoparticles.

\section{REFERENCES}

[1] Bagga, K., McCann, R., Wang, M., Stalcup, A., Vázquez, M. and Brabazon, D., "Laser assisted synthesis of carbon nanoparticles with controlled viscosities for printing applications," J. Colloid Interface Sci. 447, 263-268 (2015).

[2] Bagga, K., McCann, R., Brasi, Q., Coussy, J., Stalcup, A., Vázquez, M. and Brabazon, D., "Laser-assisted synthesis of ultrapure nanostructures for biological sensing applications," Proc. SPIE, N. Kobayashi, F. Ouchen, and I. Rau, Eds., 99280O, Nanobiosystems: Processing, Characterization, and Applications IX, San Diego, CA., USA (2016).

[3] Bagga, K., McCann, R., O’Sullivan, F., Ghosh, P., Krishnamurthy, S., Stalcup, A., Vázquez, M., Brabazon, D., Shan, C., Yang, H., Han, D., Zhang, Q., Ivaska, A., Niu, L., Riahi, R., Gogoi, P., Sepehri, S., Zhou, Y., Handique, K., et al., "Nanoparticle functionalized laser patterned substrate: an innovative route towards low cost biomimetic platforms," RSC Adv. 7(13), 8060-8069 (2017).

[4] Wagener, P., Schwenke, A., Chichkov, B. N. and Barcikowski, S., "Pulsed laser ablation of zinc in tetrahydrofuran: Bypassing the cavitation bubble," J. Phys. Chem. C 114(17), 7618-7625 (2010).

[5] Barcikowski, S. and Mafuné, F., "Trends and current topics in the field of laser ablation and nanoparticle generation in liquids," J. Phys. Chem. C 115(12), 4985 (2011).

[6] Barcikowski, S., Amendola, V., Marzun, G., Rehbock, C., Reichenberger, S., Zhang, D. and Gökce, B., [Handbook of Laser Synthesis of Colloids] (2016).

[7] Streubel, R., Barcikowski, S. and Gökce, B., "Continuous multigram nanoparticle synthesis by high-power, highrepetition-rate ultrafast laser ablation in liquids," Opt. Lett. (2016).

[8] Intartaglia, R., Bagga, K., Scotto, M., Diaspro, A. and Brandi, F., "Luminescent silicon nanoparticles prepared by ultra short pulsed laser ablation in liquid for imaging applications," 510-518 (2012).

[9] Intartaglia, R., Bagga, K. and Brandi, F., "Study on the productivity of silicon nanoparticles by picosecond laser ablation in water: towards gram per hour yield.," Opt. Express 22(3), 3117 (2014).

[10] Zhang, D., Gökce, B. and Barcikowski, S., "Laser Synthesis and Processing of Colloids: Fundamentals and Applications," Chem. Rev. 117(5), 3990-4103 (2017).

\section{ACKNOWLEDGEMENTS}

This publication has emanated from research supported the School of Biotechnology, Dublin City University and by research grants from Science Foundation Ireland (SFI) under Grant Numbers 12/IA/1576 and 16/RC/3872 and is co-funded under the European Regional Development Fund and by I-Form industry partners. The Nanoflex in-situ particle size analyser was provided on trial by Microtrac Ltd. OSL group. 